\title{
When No Laughing Matter Is No Laughing Matter: The Challenges in Developing a Cognitive Theory of Humor
}

\begin{abstract}
This paper explores the current obstacles that a cognitive theory of humor faces. More specifically, I argue that the nebulous and ill-defined nature of humor makes it difficult to tell what counts as clear instances of, and deficits in, the phenomenon. Without getting clear on this, we cannot identify the underlying cognitive mechanisms responsible for humor. Moreover, being too quick to draw generalizations regarding the ubiquity of humor, or its uniqueness to humans, without substantially clarifying the phenomenon and its occurrences is not only unwise but can actually be a detriment to our study of humor. As such, these sorts of claims must be resisted. I conclude the paper by pointing the way forward to addressing these obstacles.
\end{abstract}

Keywords: cognitive theory of humor, humor appreciation, humor comprehension, cognitive mechanisms, humor mechanism

\section{Introduction}

"What do you call a box full of ducks?"

“Quackers!”

Suppose that upon hearing this undeniably hilarious joke, your friend Joe does not laugh. We might conclude from this that Joe clearly has no sense of humor. But when should we consider that to literally be true? When does someone merely have a very different sense of humor from us, and when has the neurological mechanism responsible for the production of humor in that person broken down? Humor varies widely from era to era, culture to culture, and person to person. When is a difference of this sort indicative of a neurological or cognitive deficit in humor, and when is it not? We also know that humorous events can take many different forms. You might laugh hysterically while watching a clown get hit in the face with a pie, chuckle at a knock-knock joke, appreciate a clever political cartoon, and groan at a pun. Are all of these instances of the same type of

* University of Victoria; ehochstein@uvic.ca 
cognitive phenomenon (namely, humor)? Are some of these the product of entirely different neurological mechanisms, with entirely different evolutionary stories behind them? How can we tell?

In this paper, I argue that the nebulous and ill-defined nature of humor makes it particularly difficult to study from the perspective of cognitive science. It can be hard to tell when the phenomenon of humor is present (as opposed to a related but distinct phenomenon), and even harder to tell when there is a clear deficit in it. Without first getting clear on such matters, our ability to locate and understand the cognitive mechanisms responsible for humor becomes exceedingly difficult. This, in turn, makes it even more difficult to determine what function humor serves, how it evolved, or which creatures have it and which do not. While none of this makes the study of humor from a cognitive perspective intractable, it does suggest caution in how we proceed. Being too quick to draw generalizations about humor, or conclusions about its underlying mechanisms without substantially clarifying the phenomenon and its occurrences, is not only unwise but can actually be a detriment to our study of humor. As such, these sorts of claims must be resisted.

In order to demonstrate this, I start in section 2 by highlighting how we typically study and discover the underlying neurological mechanisms responsible for various cognitive phenomena. In section 3, I demonstrate why the nature of humor makes it particularly difficult to study in this way. In section 4, I highlight why this problem makes any generalizations about the ubiquity of humor, or our ability to identify its presence/absence in other species, unwarranted at the current time. Finally, I conclude the paper by highlighting some possible ways forward in addressing these worries.

\section{Reverse Engineering the Brain}

One of the primary goals of cognitive science is to understand how the brain does what it does to create our mental lives. In other words, it involves trying to understand how the physiological mechanisms of the brain operate so as to produce mental phenomena. Physiological mechanisms are typically defined as complex organizations of structured parts (in this case, brain regions, or collections of neurons) that are organized in very particular ways so that their inter- 
actions under specified conditions produce and sustain the relevant phenomenon. ${ }^{1}$

The study of such mechanisms often works by way of reverse engineering. Any attempt to provide a mechanistic account of a phenomenon first requires providing a detailed account of what the phenomenon is. ${ }^{2}$ This includes an interpretation of what we take the phenomenon to be, when/where the phenomenon appears, under what conditions, and what its boundaries are.

Armed with this information, we can begin to study which parts of the brain appear to be active when the phenomenon is present. Tools like PET scans, EEGs, and fMRIs allow us to determine (by way of electrical activity and blood flow) which brain regions become active when the phenomenon is present. While these studies often provide important information, and point the way towards further research, we must be cautious not to jump to conclusions about neurological mechanisms too quickly directly from such studies. First, such tools are still rather limited. They often provide very general information about brain regions, and not detailed accounts of which neural mechanisms are involved in the production of the phenomenon, or how they are operating. Second, knowing what brain activity correlates with the occurrence of the phenomenon is often insufficient by itself to indicate a causal link between such regions and the production of the phenomenon. We must supplement this information with additional accounts of when the phenomenon fails to occur as it normally should. In other words, when there is a clear deficit in the production of the phenomenon. To illustrate why this is important, consider the scientific study of memory.

Historically, it was originally thought that memory was a single unified phenomenon produced by a single cognitive mechanism. Over time, we came to learn that memory is in fact numerous different memory systems. The memory system used to remember factual information (declarative memory) is distinct from the memory system used to remember personal events (episodic memory), which is in turn distinct from phenomena like priming (implicit memory). We discovered that there was not a single memory phenomenon with a single mechanism for all memory, but in fact constituted by different memory systems produced by different sets of mechanisms. However, our ability to discover this depended on our ability to dissociate the memory systems from one another. In other words, physical damage to the brain resulted in the loss of one memory system (e.g., episodic memory), while leaving others intact (e.g., declarative

1 See Machamer, Darden and Craver 2000; Bechtel 2008; Milkowski 2013; Craver and Darden 2013; Hochstein 2016; Glennan 2017.

2 For more, see Craver and Darden 2013; Hochstein 2016. 
memory). Without being able to study when the phenomenon fails to occur as it normally would, the correlational data between the presence of the phenomenon and the underlying neurological activity can be extremely misleading.

To illustrate, suppose we still believed that all memory was the product of a single a unified mechanism. In order to discover this mechanism, we might put someone in an fMRI to observe what brain regions become active when the subject remembers something. Since I believe memory to be a unified phenomenon, I might assume that remembering the date of one's birthday (declarative memory), and remembering what happened during one's birthday (episodic memory), was the product of the same mechanism. Thus, I would look for any brain region that is active when the person engages in both kinds of activity. Because I am assuming that these are the same phenomenon, I will assume that any overlap in brain activity is clearly an indication of where the common mechanism for memory must be. But this, as we now know, would be exactly the wrong thing to conclude. The problem is that such scans will always find some overlapping brain activity in the two instances given the complex array of functions that the brain carries out simultaneously (see Van Orden and Paap 1997). Such data by itself therefore cannot disconfirm whether memory is a single unified phenomenon or not. Instead, we need to supplement this information with different kinds of information that such studies do not provide. Specifically, we need to know what happens when there is a distinct deficit in the phenomenon. It was only when subjects lost their ability to remember certain kinds of things (like reliving past events), but not others (factual information), that we suddenly had reason to question whether we were dealing with a single phenomenon or distinct phenomena. By studying subjects with deficits, we were able to more effectively study memory, and the mechanisms underlying it.

It is often when mechanisms break down that we can start to more effectively learn about their function. For another example, consider the study of vision:

The first clues as to which brain parts perform visual operations came from analyzing patients with visual deficits stemming from brain damage. Bartolomeo Panizza, who studied patients experiencing blindness after stroke-induced occipital lobe damage, proposed that the occipital lobe was the cortical center for vision. (Bechtel 2008, 91)

There are other reasons why understanding phenomena in terms of their deficits is so important. Consider a very different sort of case involving memory. Evidence has emerged that episodic memory is not what we initially thought it was. First, episodic memory is not simply the storage of past information to be recalled later, but is in fact constructive in nature. In other words, we construct our episodic memories whenever we access them. They are a type of mental sim- 
ulation that we build in order to simulate what it would be like to undergo certain events. Second, and more interestingly, such a mechanism is not strictly directed towards the past. Evidence suggests that the episodic memory mechanism is the same one that allows us to imagine ourselves in future situations, or in counter-factual circumstances. Put another way, this is not strictly a memory mechanism, but a time-traveling mental simulation mechanism, one useful for future projection as well as reliving past events (Schacter and Addis 2007; Schacter, Addis and Buckner 2007).

For our purposes, the reason this is relevant has to do with the way in which we discovered this. It turned out that subjects with brain damage that affected their episodic memory mechanism likewise affected their ability to plan for the future, or imagine themselves in different situations. The damage resulted in a deficit not only to episodic memory, but to the ability to form all kinds of mental simulations. This discovery, in turn, forced us to reconstitute what we metaphysically took the episodic memory mechanism to be.

Suppose, however, that we had no cases in which there were clear deficits in normal episodic memory functioning. In such a case, we would be unable to identify the connection between episodic memory, and other kinds of mental simulations. As such, we would have no reason to redefine our understanding of what the phenomenon is. We would continue to assume that episodic memory was focused exclusively on storing information about one's past life events, and looking for correlations between brain activity and the remembering of past events, all the while being oblivious to the connection between reliving past events and projecting oneself into future or counterfactual situations. Thus, by identifying failures in the occurrence of the phenomenon, it likewise allows us to identify deficits in other kinds of cognitive phenomena previously thought to be unrelated, and in turn forces us to radically redefine what we took the phenomenon to be.

To sum up, the study of cognitive mechanisms starts with a detailed account of what we take the phenomenon to be, and then looks both at cases where the phenomenon occurs, as well as cases where the phenomenon fails to occur, in light of both normal brain functioning and deficits in normal functioning. Only then can we make significant progress in understanding the mechanisms underlying a given phenomenon.

Before turning out attention to cognitive study of humor in particular, it is worth taking a moment to discuss the relationship between neurological theories, and cognitive theories more generally. This paper, after all, is about the problems facing the development of a cognitive theory of humor, not just a neurological one. Therefore, it is worth exploring the potential differences between these two types of theories. Neurological theories often attempt to explain phe- 
nomena by identifying and understanding the underlying neurophysiological mechanisms that produce them. Cognitive theories, on the other hand, need not appeal to any such particular neurological mechanisms. ${ }^{3}$ So does this section's focus on identifying and understanding neurological mechanisms put unfair expectations on what a cognitive theory of humor must provide?

In order to address this question, let us consider what makes something a cognitive theory to begin with. Looking at the history of cognitive science, we find that cognitive theories are often defined as such in virtue of explaining mental phenomena in terms of computation and information processing. As evidence for this claim, consider several introductory textbooks on Cognitive Science. According to Mind: Introduction to Cognitive Science, "thinking can best be understood in terms of representational structures in the mind and computational procedures that operate on those structures" (Thagard 2005, 10). Meanwhile, according to Cognitive Science: An Introduction to the Sciences of the Mind, "the most fundamental driving assumption of cognitive science is that minds are information processors" (Bermúdez 2014, 3). Or consider the International Encyclopedia of the Social and Behavioral Sciences, which says:

Cognitive modeling is the characteristic research methodology of cognitive science, resulting in theories about cognitive processes that are formulated as computer programs. A cognitive model is based on a theoretically grounded and empirically guided specification of the mental representations involved in a given cognitive function, and the computational processes operating on those representations. (Strube 2001, 2124)

If we understand cognitive theories as computational or information processing theories of mental phenomena, then we can more easily understand the driving intuition that cognitive theories may be importantly distinct from theories of neurological mechanism. Indeed, this idea has a long tradition within cognitive science (e.g., Putnam 1967; Block and Fodor 1972; Fodor 1974, 1975; Johnson-Laird 1983; Pylyshyn 1984). As José Luis Bermúdez puts it:

\footnotetext{
Many cognitive scientists have argued that cognitive processes can be studied independently of their physical realization. Just as we can understand a piece of software without knowing anything about the machine on which it runs, so too (many people have thought) we can understand cognitive processes without knowing anything about the neural machinery that runs them. (Bermúdez 2014, 60)
}

The intuition underlying this idea rests on the fact that since different hardware can run the same program or algorithm, studying an algorithm by itself does not

3 Special thanks to a blind referee for emphasizing this worry. 
tell us what hardware such an algorithm is running on. Likewise, studying the hardware of a system does not tell us what other kinds of systems may be running the same kind of programs. This suggests that a cognitive explanation (which describes the information processing task being carried out by the system) should not be conflated with a neurological one (which focuses on the implementation details of the system carrying it out).

The problem is that this intuitive distinction between cognitive and neurological theories has not withstood the test of time. Empirical factors have revealed that implementational details are essential to understanding computational facts about a given system. Moreover, our ability to test computational theories often requires a mechanistic interpretation of the system, since this is what is needed in order to manipulate the system in order to determine what functions are actually being carried out (see Eliasmith 2010). As Bermúdez puts it:

At one time cognitive science was associated with the idea that we can understand the mind without worrying about its biological machinery-we can understand the software without understanding the hardware, to use a popular image. But this is now really a minority view. Neuroscience is now an absolutely fundamental part of cognitive science. Unfortunately, this has not really been reflected in textbooks on cognitive science. (Bermúdez 2004, xxviii)

Let's consider why this is the case. The idea that we can study the cognitive features of a system independent of its implementation stems from this idea that radically different systems can realize the same computational functions. While this is true in principle, it often ignores the fact that in order for different systems to run the same program, they require different environmental conditions, and different amounts of time and resources, depending on their physical structures. This is largely because the computational architecture of a system determines what sorts of programs it is optimized to carry out efficiently under different conditions. Real world systems are always constrained by things like limited resources, limited time, and environmental pressures, which in turn means that only systems with the appropriate computational architectures will be capable of realizing those particular computational functions within those constraints. Consider the following illustrative example offered by Chris Eliasmith:

To see this, consider the example of deciding whether an object in the environment is a friend or foe. Suppose we have two different implementations of the function that needs to be computed to successfully achieve this recognition. One of these implementations, Athlon Alan, can compute this function in less than a second given its architecture, computational primitives, etc. The other implementation, Intel Alan, takes nearly 10 minutes to perform the same computation because its architecture, computational primitives, etc., aren't 
optimized for this kind of computation. In other words, the computational complexity of the algorithm on the second implementation is significantly higher than on the first implementation. Of course, if the object is a foe, Intel Alan may not have the 10 minutes required to make this decision and thus may not ever exhibit this cognitive behaviour. As a result, it is computational complexity, not function, which determines possible cognitive behaviour. Merely noting the class of abstract functions computable by Intel Alan won't tell us much about Intel Alan's actual cognitive behaviour. (Eliasmith 2002, 5)

Put simply, not any implementation of a system can carry out a given computational function given relevant limitations on time, resources, and environmental conditions. This means that knowing the neurophysiological architecture of the system becomes essential to understanding what cognitive functions it is capable of carrying out and why (for more details, see Bechtel and Mundale 1999; Eliasmith 2002, 2013; Syropoulos 2008).

It is for this very reason that some have argued that we ought to treat computational theories within cognitive science as abstract descriptions of the particular neurophysiological mechanisms that carry out the function in question; the ultimate goal of such theories being to fill in the neurological details (e.g., Piccinini 2006, 2007; Kaplan 2011; Piccinini and Craver 2011; Kaplan and Craver 2011). Even those who resist this idea, and argue that computational explanations are distinct from mechanistic explanations within cognitive science, are quick to point out that computational features of a system cannot be understood in isolation of the neurological features of that system (Chirimuuta 2014).

Likewise, our very ability to confirm or disconfirm cognitive theories often depends on our ability to manipulate the neurophysiological features of the system. Recall that it is often only when the physical mechanisms of the brain are damaged that we can get a better understanding of what functions or tasks are being carried out by the brain, and which are not. It is only with a sufficiently detailed understanding of the structural architecture of the system that we can manipulate it to see how the computational functions are affected, and thus which computational theories are accurate and which are not (Eliasmith 2010, 316).

None of this is to say that we should stop using models and theories in cognitive science that do not explicitly identify neurological mechanisms. Instead, the point is that such cognitive theories are often treated as a first step in developing a more detailed and fine-grained account of the neurological structures of the system. Since it is only by manipulating these neurological structures, or seeing how the behaviour of the system changes when such structures are damaged, that we can determine whether such theories are correct or not, and what sorts of functions are actually being carried out by the brain. Without an understanding of the computational architecture of the system, we lack essential information as 
to which sorts of algorithms or programs are physiologically plausible, and which are not.

It is for these reasons that it is important to emphasize why any cognitive theory of humor must take mechanistic features of the brain into account. It is only with an understanding of the neurological architecture, structure, and functions of the brain that we can determine which cognitive theories are plausible, and to then test them under experimental conditions. This makes such information essentially intertwined with, and not dissociable from, our cognitive theories. A cognitive theory of humor will not be divorced from a neurological story of how the brain is structured and how it operates. With all this established, let us finally turn our attention to the cognitive study of humor itself.

\section{What's So Funny about Studying Humor Mechanisms?}

The study of humor poses an interesting problem for cognitive science on the grounds that we don't have a clearly defined account of what the phenomenon is, and thus, what counts as clear instances of it, or clear deficits in its occurrence. If one groans at a pun, does this mean their humor mechanism is working properly, or has it failed miserably? Is a good turn of phrase that one finds amusing the same kind of cognitive phenomenon as the kind in which they burst out laughing watching Moe hit Curly in the nose with a hacksaw? We lump both under "humor," but are they in fact part of the same unified cognitive phenomenon, produced by the same mechanism?

Consider an episode of the animated comedy show The Simpsons. In the episode entitled "Kamp Krusty," Bart and Lisa Simpson attend a summer camp that is supposedly run by their hero, Krusty the Clown. However, Krusty is nowhere to be found. Eventually, the children at the camp are told that Krusty has come to visit, but unbeknownst to them is merely the town drunk Barney in a Krusty outfit. The children notice that "Krusty" is acting strangely, and one child at the camp remarks that "He's still funny, but not ha-ha funny." Is "funny," and "ha-ha funny" the same phenomenon? Are they the product of the same neurological mechanism? If not, is it a mistake to consider them both to be instance of the same cognitive type (humor)? Or consider an episode of the comedy series Scrubs. In the episode entitled "Her Story II," the character of JD becomes increasingly bothered by his girlfriend Julie's tendency never to laugh at jokes, but merely to enthusiastically assert "that's so funny!" in response to them. Does Julie's inability to laugh at a joke suggest an impaired sense of humor? 
Or does her declaration that the joke is hilarious show that her sense of humor is intact? On what grounds exactly are we to make this determination?

As we noted in Section 2, to understand the mechanisms underlying a cognitive phenomenon, we first must start with an understanding and conceptualization of the phenomenon, then attempt to identify cases of its occurrence, as well as cases in which there are clear deficits in the phenomenon. With this information, we can start to localize the mechanisms responsible for it. When it comes to humor, each of these runs into serious obstacles. Let's begin with conceptualization:

In order to study the mechanisms underlying a phenomenon, we first must start with a conceptualization of what we take the phenomenon to be (for details, see Hochstein 2019). Yet with humor, this is more difficult that it appears. Humor seems to come in all shapes and sizes, encompassing everything from political cartoons, to knock-knock jokes, to puns, to slapstick, to subtle satire, to making fart noises. To complicate matters further, someone may find fart noises incredibly funny, while someone else finds them childish and immature. What counts as humor appears to vary wildly depending on the era, the individual, and the culture one is a part of. This makes it unclear exactly how we ought to define humor. As Wild et al. (2003) rightly points out:

\footnotetext{
Reasons for the complexity of research on humour are legion. What was funny 20 years ago may not be funny today and the meanings of such terms as 'humour', 'funny', 'mirth' and 'hilarity' vary not only with time but also among languages and cultures. [...] Although operational definitions of 'laughter,' 'humour' and 'funny' have been formulated for individual studies, a broad consensus on their exact meanings has yet to be reached. This is not a trivial handicap: it is obvious that what one means by humour and laughter will influence what kinds of experiments one designs for their analysis. (Wild et al. 2003, 2131)
}

This worry is likewise echoed by Olin, who argues that our concept of humor is "promiscuous," and that it is "used to refer to an enormous range of things that are perceived as funny" (Olin 2016, 338).

One way that theorists have tried to make progress in the face of such diversity is by identifying features that all cases of humor seem to have in common. Specifically, all instances of humor (from puns to political cartoons) appear to involve humor comprehension (our ability to get the joke), humor appreciation (the affective experiential component of finding something funny), and the behavioral response to humor (laughing, chuckling, etc.). In the example of Julie from Scrubs mentioned earlier, her ability to understand that a joke is hilarious, but not to laugh at it, may be an indication that her humor comprehension is working fine, but either her humor appreciation, or her behavioral response to humor, may not be working properly. 
While this intuitive 3-part account of humor is widely appealed to in the literature $^{4}$, adopting this framework can make the phenomenon of humor seem deceptively more well-defined than it in fact is. For instance, humor appreciation is often characterized as "moments where the perceiver experiences pure visceral, emotional responses dependent upon the hilarity of the experience" (Moran et al. 2004, 1055). Yet there are many different kinds of visceral emotional responses one might have to a joke that are completely unrelated to "the hilarity of the experience” (e.g., fear, sadness, confusion, etc.). And so, what tells us that a particular emotional response is one of hilarity as opposed to some other kind of emotional state? The implicit assumption is that humor experiences (often referred to as "mirth" in the literature) is a particular kind of experience that all instances of genuine humor share. Yet what exactly counts as a state of mirth remains extremely unclear, and not nearly as well defined or straightforward as it seems. Appreciating a good turn of phrase is phenomenologically a very different experience from busting a gut laughing at a fart joke. The danger of assuming that these different affective states are all of the same type (namely, humor experiences) is that we may be using our folk conception of humor to interpret our affective states, instead of identifying any sort of natural kind similarity between the states themselves. In other words, we are not so much classifying preexisting types of mirth-states as we are conforming our interpretation of our affective states to fit with our folk category of "humor" (i.e., we interpret a state as mirth because of how we use the folk concept of humor).

One can see the danger in this by examining a parallel case in the study of emotion. It may be equally tempting to argue that the affective component to an emotion like anger is how we can tell that different instances of anger are all instances of the same type of emotion. But evidence has increasingly shown that "anger" is not a single type of emotion. Instead, the folk concept of "anger" refers to a range of distinct and unrelated cognitive processes, produced by distinct cognitive mechanisms, with different evolutionary origins (see Griffith 1997, 2004).

Lisa Feldman Barrett (2017) likewise argues that we interpret our emotional experiences through the linguistic categories we've been taught. And so various experiential states will be classified as "anger" not because they share any sort of deep phenomenological similarity or mechanistic underpinning, but because the way in which we've been taught to apply the "anger" concept directly influences how we make sense of our own experiences. The illusion that we can all

4 E.g., Adamczyk et al. 2017; Bambini et al. 2020; Bischetti et al. 2019; Mobbs et al. 2003; Rozengurt 2011; Samson 2013; Samson and Hegenloh 2010; Chen et al. 2017. 
easily recognize anger when we experience it comes from the fact that our experiences are structured by, and filtered through, our emotion concepts. Thus, we force our experiences to fit into the categories we provide, instead of recognizing some fundamental similarity between different experiential states. Given how much humor varies from culture to culture, this might very well be true of the concept of "humor" as well. The singular term "humor appreciation" may obscure the fact that there may be many different kinds of distinct and unrelated mechanisms and processes responsible for the affective component of different cognitive states that we have mistakenly treated as features of the same category.

Next, consider that what counts as "humor comprehension" is similarly unclear. As Mak and Carpenter note, "the cognitive underpinnings of [humor] comprehension are not understood well enough to make strong claims regarding its interaction with subsequent processes" $(2007,606)$. One of the dominant views in the field is that humor comprehension involves the identification, and resolution, of incongruous elements within a given joke (e.g., Raskin 1985; Morreall 1987; Richie 1999; Samson 2013). As Olin puts it:

The funny, on this view, is found in the incongruous: we laugh at things that are unexpected or unusual because they surprise us, or seem somehow out of place. The experience of being amused can be described in terms of the recognition of the fact that you were thinking about something in a different way, or from a different perspective, than that called for by the overall context. (Olin 2016, 343)

As a possible theory of humor comprehension, this is certainly plausible. But acceptance of this theory will depend on exactly how we define incongruity and resolution, as well as whether we can identify clear instances of incongruity in cognitive reasoning more generally, as well as clear deficits in its occurrence, in order to identify and understand the underlying mechanisms for it. Then, we can observe whether this mechanism is similarly active in cases of humor processing in particular (since if it turns out that this mechanism plays no part in many cases of humor, then this would suggest that many instances of humor comprehension does not involve incongruity after all, even if we can superficially describe them as though they do). To adopt this theory too early would be again to make the phenomenon of humor seem far more well-defined than it is.

If, for instance, we start with the assumption that incongruity is a necessary condition for humor (as many theorists do), then potentially genuine cases of humor that do not involve incongruity will be dismissed or treated as outliers, distorting the results of our experiments. Put simply, being too quick to adopt such a definition of humor comprehension without the relevant neurological data can subtly shift how we scientifically study the phenomenon of humor, 
and humor comprehension. Subtly different definitions or understandings of what counts as humor, humor comprehension, and humor appreciation, can radically influence the sorts of experiments and methodologies that scientists employ to study it. An experiment that may yield results when humor is understood one way, may be completely uninformative when understood in a subtly different way. These sorts of definitional and categorizational problems must be taken seriously, as proceeding incautiously here can pull us down the wrong road from the start. ${ }^{5}$

But perhaps we don't need a clear definition of humor in order to effectively study it from a cognitive perspective, just so long as we can reliably identify the phenomenon's occurrence. Vrticka, Black and Reiss, for example, claim that "despite such multiple usages and definitions of humor, nearly all of us can easily recognize humor when we experience it" $(2013,860)$. Moreover, our ability to recognize different experiences as belonging to the same type (humor) might likewise suggest that humor is a unified phenomenon with a single underlying mechanistic cause. Ramachandran, for example, draws exactly this conclusion when he insists that:

The details of the phenomenon vary from culture to culture and are influenced by the way you were raised, but this does not mean that there is no genetically specified mechanism for laughter, a common denominator underlying all types of humour. Indeed, many people have suggested that such a mechanism does exist and theories on the biological origins of humour and laughter have a long history, going all the way to Schopenhauer and Kant, two singularly humourless German philosophers. (Ramachandran 1998, 1857)

This brings us to the second set of obstacles facing the cognitive study of humor: identifying instances of its occurrence. To claim that we can all easily recognize humor when we experience it is extremely misleading. How exactly would we be able to tell if we had an experience of humor but never recognized it as such? Or if we recognized an experience as being humor when it wasn't? What could we use as a guide for the correct or incorrect recognition and classification of our own experiences? Indeed, the cases of misclassification might greatly outnumber the cases in which we do easily recognize humor when we experience it. Worse still, the fact that we tend to classify experiences as "humor" does not, by itself, tell us anything about whether the various experiences form a natural kind, or are the product of the same mechanism. Here again the analogy to anger is informative. We might likewise claim that we can all easily recognize anger when we experience it. Yet, this does not in fact show that anger is a natural kind, and may in-

5 For more details on this problem, see Hochstein 2019. 
stead show only that the anger concept influences how we classify our affective states (Barrett 2017).

But for the moment, let's assume that these obstacles to identifying instances of the phenomenon do not apply. Let us, for the sake of argument, assume that we can identify a few uncontroversial instances of humor. From there, it is tempting to assume that we can directly study the mechanisms for humor by trying to correlate brain activity with the occurrence of a humorous event. In such cases, we might put the subject in an fMRI, or another type of brain-scanning instrument, and observe what brain regions become active when they are presented with visual or linguistic jokes. ${ }^{6}$ The problem with such studies, as we noted in Section 2, is that such correlational data by itself is extremely limited. This problem is exacerbated by the fact that we can't tell whether the concept of "humor" denotes a unified cognitive phenomenon to begin with or not. Thus, to look for any shared neural activity in all the cases we deem to be "humor" in order to declare it part of the underlying mechanism for humor is hugely problematic. But this is exactly what many have been tempted to do. Consider the following passage from Watson, Matthews and Allman (2007) who, despite noting that two different brain networks seemed to be activated by two different kinds of humor events, still insist there is a shared mechanism by looking at any overlap in the two cases:

We used event related functional magnetic resonance imaging to differentiate brain activity
induced by the hedonic similarities and cognitive differences inherent in 2 kinds of humor:
visual humor (sight gags) and language-based humor. Our findings indicate that the brain
networks recruited during a humorous experience differ according to the type of humor
being processed, with high-level visual areas activated during visual humor and classic lan-
guage areas activated during language-dependent humor. Our results additionally highlight
a common network activated by both types of humor that includes the amygdala and mid-
brain regions, which presumably reflect the euphoric component of humor. (Watson, Mat-
thews and Allman 2007, 314)

Yet this is exactly what one should not conclude from such correlational data. Without a far greater degree of clarity in terms of what we mean by "humor," and clear cases of deficits in its production, making any sort of claim about shared underlying mechanisms is unfounded. This is not to say that such correlational data is not relevant or important. Only that we must be extremely cautious about what can, and about what cannot, be inferred from such information

6 For examples of just such studies, see Derks et al. 1997; Ozawa et al. 2000; Coulson and Kutas 2001; Iwase et al. 2002; Mobbs 2003; Moran et al. 2004; Watson, Matthews and Allman 2007; McGettigan et al. 2015; Chen et al. 2017; Chan et al. 2018; Hofmann and Rodden 2019. 
alone. And the temptation to infer more than is warranted from these studies has been a serious obstacle to progress.

Lastly, consider attempts to identify deficits in humor. What exactly does a deficit in humor look like? If one finds a fart joke distasteful, while another finds it hilarious, do either one of them have a deficit in their humor mechanism (e.g., in their humor appreciation or comprehension)? If so, which one? If not, then how can we tell when there is a clear deficit in the production of humor, or when people just have different senses of humor? Laughter itself seems an insufficient guide here, as laughter can be caused by all kinds of events that have nothing to do with humor. Moreover, the use of pathological laughter for determining deficits in humor won't help either, as "the notion of 'pathological' laughter can refer to anything from laughter at politically incorrect jokes to laughter as a manifestation of chromosomal aberrations in the Angelman syndrome" (Wild et al. 2003, 2122). Here again we see how conceptual confusion surrounding humor can directly influence whether we count something as a deficit in humor or not.

Or consider a number of studies that claim to show a deficit in humor for those with conditions like autism spectrum disorder (Samson and Hegenloh 2010; Samson 2013), schizophrenia (Vrticka, Black and Reiss 2013; Adamczyk et al. 2017), amyotrophic lateral sclerosis (Bambini et al 2020), alcohol use disorder (Uekermann et al. 2007; Le Berre, Fama, and Sullivan 2017), and major depression (Uekermann et al. 2008). To understand these cases, one must understand the role that Theory of Mind is thought to play in many kinds of jokes. Theory of Mind (ToM) is a psychological phenomenon in which we are able to put ourselves in the shoes of others, either by way of attributing mental states to others that we ourselves do not presently have, or by way of mentally simulating what it would be like to be them (see Premack and Woodruff 1978; Wellman 2017). Studies have suggested that a breakdown in the ToM mechanism seems to result in a humor deficit in the disorders mentioned above, pointing to possible underlying mechanisms for humor:

Behavioural, imaging and brain lesion findings associated with humour and psychopathology have also been published. For example, one line of research reports deficient humour processing in autism. These results are linked to the individual's difficulties in understanding the social aspect of humour requiring ToM and, more generally, in integrating cognitive and affective information. [...] Other research indicates impaired humour appreciation in schizophrenia. Affected individuals display reduced humour recognition associated with impaired ToM abilities and diminished $\mathrm{mPFC}$ activity in response to jokes requiring the attribution of mental states. Therefore, in both autism and schizophrenia, humour appreciation deficits seem to be predominantly related to disturbed ToM and/or socioemotional in- 
tegration mechanisms, and thus cognitive humour processing. (Vrticka, Black and Reiss 2013, 866)

But again, extreme care and caution must be taken here. While people with schizophrenia, autism spectrum disorder, and other psychiatric conditions may have difficulty with ToM, and this may make it difficult for them to comprehend certain kinds of jokes or humorous events, it is not at all apparent that this demonstrates that they have a deficit in humor. For instance, not all jokes depend on ToM (e.g., Samson, Zysset and Huber 2008; Samson and Hegenloh 2010), and if their sense of humor remains intact in those situations, then the problem may not be with their sense of humor, and only with the ToM needed to appreciate certain kinds of jokes. This is suggested by Samson and Hegenloh's findings that individuals with autism "did not differ to the control group in humor appreciation of visual puns. However, they had difficulty understanding and appreciating Theory of Mind cartoons" $(2010,438)$. One possibility here is that the humor module (if there is such a thing) may function perfectly in those with autism spectrum disorder, schizophrenia, and any other psychiatric condition that inhibits ToM. But their deficits in ToM mean that certain kinds of mental state attributions needed as inputs to the humor module for certain jokes to be understood are not available. As such, they will have difficulty with humor comprehension with those jokes in particular. But not due to any sort of breakdown in the mechanisms that produces and sustains a sense of humor. In the same way that someone who is blind may be unable to identify or appreciate certain kinds of visual gags, but it would hardly follow that blindness results in a humor deficit, or humor comprehension deficit. A fairly well-known joke can help illustrate the potential worry here:

In an effort to better understand frog physiology, a scientist trains a healthy four-legged frog to jump on command. The scientist then tries to see how the abilities of the frog are hindered when its limbs are removed. She first removes one leg, and then instructs the frog to jump. It does. The scientist notes in her log that the frog can successfully jump with three legs. She then removes the second leg, and instructs the frog to jump again. It does. The scientist notes in her log that the frog can jump with just two legs. She then removes the third leg. Upon witnessing the frog jump on command a third time, she notes that the frog can still jump even with a single leg. Finally, she removes the frog's last leg, and instructs it to jump. The frog does not move. Upon seeing this, she notes in her log "upon having all four legs removed, frog becomes deaf."

The point is that the lack of a clear definition of humor, and the fact that humor takes many different forms, makes it difficult to tell when neurological damage results in a humor deficit, or a deficit in some other type of phenomen- 
on. If something affects our ability to appreciate certain kinds of humor, but not others, does this show that there is a deficit in humor or not? Are there different humor mechanisms that are unrelated (just as there are different memory systems)? If so, what makes them all instances of the same type of phenomenon, as opposed to distinct kinds of phenomena that we have mistakenly all labelled "humor"? Again, confusion surrounding how we define humor and what counts as instances of it provides serious obstacles to identifying what exactly a deficit in humor would look like, and whether what seems like a deficit in humor may in fact be a deficit in something else.

With these sorts of obstacles facing the cognitive study of humor, one would expect caution with the sorts of scientific generalizations one makes regarding humor. Being too quick to jump to conclusions about underlying mechanisms, their functions, and their evolutionary origins, can cause even greater confusion in the study of humor by assuming the phenomenon is more well defined than it is, or by smuggling in unjustified assumptions about its occurrences and deficits. And yet this has been surprisingly common in the cognitive literature, adding to the obstacles that a cognitive theory of humor must now overcome. It is to these concerns that we turn to next.

\section{Jumping the Gun}

Given the complications surrounding the way we define humor, our ability to identify its occurrences and deficits, and to locate its underlying causal mechanisms, we must be cautious about the sorts of generalizations we make about humor. The temptation to assume that the phenomenon is more well defined than it is, or that we can clearly identify its presence or absence in contentious contexts, must be resisted if we are to proceed responsibly. To illustrate the sorts of dangers that such temptations offer, consider the following cases.

First, consider Roman Rozengurt's claim that "Humor is a very interesting phenomenon in human cognitive and emotional processing, and it is unique for human beings" (2011, 71). Or Moran et al.'s insistence that "humor is a uniquely human quality whose neural substrates remain enigmatic" (2004, 1055). If the neural substrates of humor remain enigmatic, and the phenomenon itself is not clearly defined, then what grounds do we have for confidently asserting that humor is unique to humans? Some have argued that depending on how we understand and define humor, it may well be present in many non-human animals (see Gramble 2001; Panksepp 2005; Beakoff 2007). Gramble, for instance, argues that if we adopt the incongruity theory of humor, then evidence does show that other great apes do experience humor (Gramble 
2001). Similarly, McGhee argues that the conditions we use to identify humor in young children provide strong evidence for the existence of humor in other animals. Specifically, Paul McGhee says:

... it is clear that nothing that an animal could do would constitute unequivocal proof of a humor experience. But, granted the observation of the three conditions just described for children, we would be safe in concluding that the animal has met all of the necessary prerequisites for experiencing humor. (McGhee 1979, 96)

This means that substantially more work is required to clearly define and identify humor before we can responsibly make claims about its uniqueness in humans. Moreover, if we assume that humor is unique to humans, this can unduly influence how we think about the phenomenon, and thus how we test for it. Differences between human brains and animal brains can easily become the foundation for trying to locate the mechanisms for humor in the brain regions only found in humans. And yet if such an assumption turns out to be false, then we would not only be looking in the wrong place, but may dismiss genuine instances of the phenomenon in non-human animals because we are convinced the phenomenon cannot apply to them. Until we understand more about the underlying neural structures responsible for humor in humans, we cannot confidently dismiss its presence in other animals.

Or consider claims regarding the ubiquity of humor within our own species. Watson, Matthews and Allman claim that "The phenomenon of humor is universal among humans." (2007, 314). Meanwhile, Brownell and Stringfellow insists that "All humans have the ability to appreciate humor; everyone can laugh and experience mirth" (2002, 241). But again, given a lack of information regarding the mechanisms for humor, and a clear definition of humor to work with, such claims can be difficult if not impossible to justify. Recall that there are numerous studies that appear to show that certain psychiatric conditions (like major depression, and schizophrenia, among others) result in a deficit in humor. If such studies are correct, it would clearly suggest that humor is not a universal component of human experience, nor that all humans have the ability to appreciate humor.

What are we to conclude about the ubiquity of humor in human life? At the moment, until more careful work is done, we must be careful not to conclude anything. This is not to say that no such answer is forthcoming, but only that suggesting we already have such answers in hand gives the misleading impression that humor is more well defined than it is, or that its occurrences and deficits are easily detectable when they are not. 
All of these cases demonstrate the potential dangers in being too quick to make generalizations about humor before we do our requisite homework. Caution is required as we attempt to disentangle our different conceptualizations of humor and develop more clear methods for identifying its occurrence and deficits. Once this is done, we will be able to make more substantial progress in uncovering and understanding the underlying mechanisms responsible for the phenomenon. Then, and only then, can we start making more generalized claims about humor's ubiquity, and presence across species that are justified by the relevant evidence.

\section{Conclusion: Finding a Way Forward}

I have spent a great deal of time highlighting the obstacles to developing a cognitive account of humor. However, it is worth ending the paper on a more optimistic note. None of the obstacles discussed here are scientifically intractable, nor do they suggest that we cannot develop a well-grounded cognitive theory of humor. After all, the problems that a cognitive theory of humor faces are hardly unique. As Wild et al. (2003) point out...

... the notions of laughter and humour are no more intractable now than crying and pain once were. Indeed, the latter pair of phenomena share important characteristics with humour and laughter. Crying and pain are also mixtures of subjectivity, neurology and poetry. Despite these confounds, however, so much has been learned about pain and its expressions over the past 100 years that a review of their neural correlates would occupy a small encyclopaedia. (Wild et al. 2003, 2131)

This gives us encouraging reasons to think that the study of humor will also make great strides over the coming decades. But just like the study of humor, it would have been equally as irresponsible for people in the past to have confidently asserted that pain was a uniquely human characteristic, or to have posited a single mechanism for all phenomena we subsume under the category of "pain" (be it physical inquiry, emotional distress, depression, etc.). The way forward in studying humor, as it was with pain, requires paying close attention to the subtleties of how the phenomena is defined and understood, and the methodological complications that identifying its occurrences face, and not to be quick to gloss them over.

What is required is care and patience in how we proceed. Neurological theories regarding the organizational structure of the brain (and whether it is modular or more deeply interconnected in nature) can help to determine if there even are any dedicated humor mechanisms or modules we should search for or not, 
and what sorts of experiments would yield informative outcomes as a result (for more, see Patterson and Plaut 2009; Anderson 2010; Eliasmith 2013).

Linguistic and philosophical theories which provide a conceptual analysis of how the term "humor" is used in different contexts and cultures can help to clarify how we think about the phenomenon, and how different ways of thinking about the phenomenon may influence or structure our experience or feeling of mirth (Yue et al. 2016; Barrett 2017; Jiang, Li and Hou 2019).

Psychological and cognitive theories of humor like relief theories (Spencer 1860; Freud 1905), superiority theories (Morreall 1983, 4-5), incongruity theories (Raskin 1985; Morreall 1987; Richie 1999; Samson 2013), and others (Olin 2016; Olin 2020) need to be further developed to see whether any can satisfactorily account for the whole range of events and experiences treated as humorous across cultures and individuals. Their success or failure in this regard can help pave the way for whether we should think of all instances of humor as a single unified type of cognitive phenomenon, or as a collection of distinct cognitive mechanisms.

This means that we have a way forward in the cognitive study of humor so long as we proceed responsibly, and resist the temptation to make grand claims when the evidence does not allow for it. In this regard, the study of humor is no laughing matter; unless of course it involves a joke about quackers. Then it is hilarious.

\section{References}

Adamczyk, Przemysław, Miroslaw Wyczesanyc, Aleksandra Domagalikd, Artur Daren, Kamil Cepuch, Piotr Błądziński, Andrzej Cechnicki, and Tadeusz Marek. 2017. "Neural Circuit of Verbal Humor Comprehension in Schizophrenia-an fMRI Study." Neurolmage: Clinical 15: $525-40$.

Anderson, Michael. 2010. "Neural Reuse: A Fundamental Organizational Principle of the Brain." Behaviorial and Brian Science 33: 245-313.

Bambini, Valentina, Luca Bischetti, Chiara Giuseppina Bonomi, Giorgio Arcara, Serena Lecce, and Mauro Ceroni. 2020. "Beyond the Motor Account of Amyotrophic Lateral Sclerosis: Verbal Humour and its Relationship with the Cognitive and Pragmatic Profile." INT J LANG COMMUN DISORD 55(5): 751-64.

Barrett, Lisa Feldman. 2017. How Emotions Are Made. Boston, MA: Houghton Mifflin Harcourt.

Beakoff, Marc. 2007. The Emotional Lives of Animals: A Leading Scientist Explores Animal Joy, Sorrow, and Empathy - and Why They Matter. Novoto, CA: New World Library. Bechtel, William, and Jennifer Mundale. 1999. "Multiple Realizability Revisited: Linking Cognitive and Neural States." Philosophy of Science 66(2): 175-207. 
Bechtel, William. 2008. Mental Mechanisms: Philosophical Perspectives on Cognitive Neuroscience. New York: Lawrence Erlbaum Associates.

Bermúdez, José Luis. 2014. Cognitive Science: An Introduction to the Science of the Mind. $2^{\text {nd }}$ edition. New York, NY: Cambridge University Press.

Bischetti, Luca, Irene Ceccato, Serena Lecce, Elena Cavallini, and Valentina Bambini. 2019. "Pragmatics and Theory of Mind in Older Adults' Humor Comprehension." Current Psychology. https://doi.org/10.1007/s12144-019-00295-w

Block, Ned, and Jerry Fodor. 1972. "What Psychological States Are Not.” The Philosophical Review 81(2): $159-81$.

Brownell, Hiram, and Andrew Stringfellow. 2002. "Cognitive Perspectives on Humor Comprehension after Brain Injury." In Neurobehavior of Language and Cognition, edited by L.T. Connor and L.K. Obler. 241-58. Boston, MA: Springer.

Chen, Hsueh-Chih, Yu-Chen Chan, Ru-Huei Dai, Yi-Jun Liao, and Cheng-Hao Tu. 2017. "Neurolinguistics of Humor." In The Routledge Handbook of Language and Humor, edited by S. Attardo. 282-94. New York, NY: Routledge Handbooks.

Chan, Yu-Chen, Wei-Chin Hsu, Yi-Jun Liao, Hsueh-Chih Chen, Cheng-Hao Tu and Ching-Lin Wu. 2018. "Appreciation of Different Styles of Humor: An fMRI Study." Scientific Reports 8 (15649).

Chirimuuta, Mazviita. 2014. "Minimal Models and Canonical Neural Computations: The Distinctness of Computational Explanation in Neuroscience." Synthese 191(2): 127-54.

Coulson, Seana, and Marta Kutas. 2001. "Getting it: Human Event-Related Brain Response to Jokes in Good and Poor Comprehenders." Neuroscience Letters 316: 71-4.

Craver, Carl, and Lindley Darden. 2013. In Search of Mechanisms. Chicago, IL: University of Chicago Press.

Derks, Peter, Lynn Gillikin, Debbie Bartolome-Rull, and Edward Bogart. 1997. "Laughter and Electroencephalographic Activity." Humor 10(3): 285-300.

Eliasmith, Chris. 2002. "The Myth of the Turing Machine." Journal of Experimental and Theoretical Artificial Intelligence 14: 1-8

Eliasmith, Chris. 2010. "How we Ought to Describe Computation in the Brain." Studies in History and Philosophy of Science Part A 41: 313-20.

Eliasmith, Chris. 2013. How to Build a Brain: A Neural Architecture for Biological Cognition. Oxford: Oxford University Press.

Fodor, Jerry. 1974. "Special Sciences (or: The Disunity of Science as a Working Hypothesis)." Synthese 28(2): 97-115.

Fodor, Jerry. 1975. The Language of Thought. Cambridge, MA: Harvard University Press.

Freud, Sigmund. 1905/1989. Jokes and Their Relation to the Unconscious. New York, NY: W.W. Norton and Co.

Glennan, Stuart. 2017. The New Mechanical Philosophy. Oxford: Oxford University Press.

Gramble, Jennifer. 2001. “Humor in Apes.” Humor 14(2): 163-79.

Griffiths, Paul. 1997. What Emotions Really Are: The Problem of Psychological Categories. Chicago, IL: University of Chicago Press.

Griffiths, Paul. 2004. "Emotions as Natural and Normative Kinds.” Philosophy of Science 71(5): 901-11.

Hochstein, Eric. 2016. "One Mechanism, Many Models: A Distributed Theory of Mechanistic Explanation.” Synthese 193: 1387-407. 
Hochstein, Eric. 2019. "How Metaphysical Commitments Shape the Study of Psychological Mechanisms." Theory and Psychology 9(5): 579-600.

Hofmann, Jennifer, and Frank A. Rodden. 2019. "Representing, Detecting, and Translating Humour in the Brain." In The Handbook of the Neuroscience of Multilingualism, edited by J.W. Schwieter and M. Paradis. 335-54. Hoboken, NJ: Wiley.

Iwase, Masao, Yasuomi Ouchi, Hiroyuki Okada, Chihiro Yokoyama, Shuji Nobezawa, Etsuji Yoshikawa, Hideo Tsukada, Masaki Takeda, Ko Yamashita, Masatoshi Takeda, Kouzi Yamaguti, Hirohiko Kuratsune, Akira Shimizu, and Yasuyoshi Watanabe. 2002. "Neural Substrates of Human Facial Expression of Pleasant Emotion Induced by Comic Films: A PET Study.” Neuroimage 17: 758-68.

Jiang, Tonglin, Hao Li, and Yubo Hou. 2019. “Cultural Differences in Humor Perception, Usage, and Implications." Frontiers in Psychology 10: 123.

Johnson-Laird, Philip. 1983. Mental Models: Towards a Cognitive Science of Language, Inference and Consciousness. New York, NY: Cambridge University Press.

Kaplan, David Michael. 2011. "Explanation and Description in Computational Neuroscience." Synthese 183: 339-73.

Kaplan, David Michael, and Carl Craver. 2011. "The Explanatory Force of Dynamical and Mathematical Models in Neuroscience: A Mechanistic Perspective." Philosophy of Science 78(4): 601-27.

Le Berre, Anne-Pascale, Rosemary Fama, and Edith Sullivan. 2017. "Executive Functions, Memory, and Social Cognitive Deficits and Recovery in Chronic Alcoholism: A Critical Review to Inform Future Research." Alcoholism: Clinical and Experimental Research 41 (8): $1432-43$.

Machamer, Peter, Lindley Darden, and Carl Craver. 2000. "Thinking About Mechanisms.” Philosophy of Science 67(1): 1-25.

Mak, Wingyun, and Brian D. Carpenter. 2007. "Humor Comprehension in Older Adults." Journal of the International Neuropsychological Society 13(4): 606-14.

McGettigan, Carolyn, E. Walsh, R. Jessop, Z. K. Agnew, D. A. Sauter, J. E. Warren, and S. K. Scott. 2015. "Individual Differences in Laughter Perception Reveal Roles for Mentalizing and Sensorimotor Systems in the Evaluation of Emotional Authenticity." Cerebral Cortex 25(1): $246-57$.

McGhee, Paul. 1979. Humor: Its Origin and Development. San Francisco, CA: W. H. Freeman and Co.

Milkowski, Marcin. 2013. "A Mechanistic Account of Computational Explanation in Cognitive Science." In Cooperative Minds: Social Interaction and Group Dynamics. Proceedings of the 35th Annual Meeting of the Cognitive Science Society, 3050-5. Cognitive Science Society.

Mobbs, Dean, Michael Greicius, Eiman Abdel-Azim, Vinod Menon, and Allan Reiss. 2003. "Humor Modulates the Mesolimbic Reward Centers." Neuron 40(5): 1041-8.

Moran, Joseph, Gagan Wig, Reginald Adams Jr., Petr Janata, and William Kelley. 2004. "Neural Correlates of Humor Detection and Appreciation." Neurolmage 21: $1055-60$.

Morreall, John. 1983. Taking Laughter Seriously. Albany, NY: State University of New York Press.

Morreall, John, ed. 1987. The Philosophy of Laughter and Humor. Albany, NY: State University of New York Press.

Olin, Lauren. 2016. "Questions for a Theory of Humor.” Philosophy Compass 11(6): 338-50. 
Olin, Lauren. 2020. “The Comic Stance.” The Philosophy of Humor Yearbook 1(1): 49-71.

Ozawa, Fukujiro, Kayako Matsuo, Chikako Kato, Toshiharu Nakai, Haruo Isoda, Yasuo Takehara, Tetsuo Moriya, and Harumi Sakahara. 2000. "The Effects of Listening Comprehension of Various Genres of Literature on Response in the Linguistic Area: An fMRI Study." Neuroreport 11: 1141- 43.

Panksepp, Jaak. 2005. "Beyond a Joke: From Animal Laughter to Human Joy?” Science 308: 62-3.

Patterson, Karalyn, and David Plaut. 2009. "Shallow Draughts Intoxicate the Brain: Lessons From Cognitive Science for Cognitive Neuropsychology." Topics in Cognitive Science 1(1): 39-58.

Piccinini, Gualtiero. 2006. “Computational explanation in neuroscience." Synthese 153: 343-53.

Piccinini, Gualtiero. 2007. “Computing mechanisms.” Philosophy of Science 74(4): 501-26.

Piccinini, Gualtiero, and Carl Craver. 2011. "Integrating Psychology and Neuroscience: Functional Analyses as Mechanism Sketches." Synthese 183(3): 283-311.

Premack, David, and Guy Woodruff. 1978. "Does the Chimpanzee Have a Theory of Mind?" Behavioral and Brain Sciences 1: 515-26.

Putnam, Hilary. 1967. "Psychological Predicates." In Art, Mind, and Religion, edited by W.H. Capitan and D.D. Merrill. 37-48. Pittsburgh, P.A.: University of Pittsburgh Press.

Pylyshyn, Zenon W. 1984. Computation and Cognition. Cambridge, MA: MIT Press.

Ramachandran, Vilayanur Subramanian. 1998. "Consciousness and Body Image: Lessons From Phantom Limbs, Capgras Syndrome and Pain Asymbolia.” Phil. Trans. R. Soc. Lond. B 353(1377): 1851-59.

Raskin, Victor. 1985. "Semantic Mechanisms of Humor." In Semantic Mechanisms of Humor, Synthese Language Library, vol. 24. Dordrecht: D. Reidel.

Ritchie, Graeme. 1999. "Developing the Incongruity-Resolution Theory." Proceedings of the AISB 99 Symposium on Creative Language: Humour and Stories, 78-85.

Rozengurt, Roman. 2011. "Neuroscience of Humor Processing: A Selective Review." Humor Mekuvvan: Research Journal in Humor Studies 1: 72-8.

Samson, Andrea Christiane. 2013. "Humor(lessness) Elucidated - Sense of Humor in Individuals with Autism Spectrum Disorders: Review and Introduction." Humor 26(3): 393-409.

Samson, Andrea Christiane, and Michael Hegenloh. 2010. "Stimulus Characteristics Affect Humor Processing in Individuals with Asperger Syndrome." J Autism Dev Disord 40: 438- 47.

Samson, Andrea Christiane, Stefan Zysset, and Oswald Huber. 2008. "Cognitive Humor Processing: Different Logical Mechanisms in Nonverbal Cartoons-An fMRI Study." Social Neuroscience 3(2): 125-40.

Schacter, Daniel, and Donna Rose Addis. 2007. "The Ghosts of Past and Future: A Memory That Works by Piecing Together Bits of the Past May be Better Suited to Simulating Future Events Than One That is a Store of Perfect Records." Nature 445(7123): 27.

Schacter, Daniel, Donna Rose Addis, and Randy Buckner. 2007. "Remembering the Past to Imagine the Future: The Prospective Brain.” Nat Rev Neurosci 8: 657-61.

Spencer, Herbert. 1860/1911. "The Physiology of Laughter." In Essays on Education and Kindred Subjects. London: Dent. 
Strube, Gerhard. 2001. "Cognitive Modeling: Research Logic in Cognitive Science." International Encyclopedia of the Social and Behavioral Sciences, 2125-8.

Syropoulos, Apostolos. 2008. Hypercomputation: Computing Beyond the Church-Turing. Barrier: Springer.

Thagard, Paul. 2005. Mind: Introduction to Cognitive Science. $2^{\text {nd }}$ edition. Cambridge, MA: The MIT Press.

Uekermann, Jennifer, Shelley Channon, Katja Winkel, Peter Schlebusch, and Irene Daum. 2007. "Theory of Mind, Humour Processing and Executive Functioning in Alcoholism." Addiction 102: 232- 40.

Uekermann, Jennifer, Shelley Channon, Caroline Lehmkämper, Mona Abdel-Hamid, Wolfgang Vollmoeller, and Irene Daum. 2008. "Executive Function, Mentalizing and Humor in Major Depression.” Journal of the International Neuropsychological Society 14(1): 55-62.

Van Orden, Guy, and Kenneth R. Paap. 1997. "Functional Neuroimages Fail to Discover Pieces of Mind in the Parts of the Brain." Philosophy of Science 64: S85-S94.

Vrticka, Pascal, Jessica M. Black, and Allan L. Reiss. 2013. "The Neural Basis of Humour Processing." Nat Rev Neurosci 14: 860-8.

Watson, Karli, Benjamin J. Matthews, and John M. Allman. 2007. "Brain Activation During Sight Gags and Language-Dependent Humor." Cerebral Cortex February 17: 314-24.

Wellman, Henry M. 2017. "The Development of Theory of Mind: Historical Reflections." Child Development Perspectives 11(3): 207-14.

Wild, Barbara, Frank A. Rodden, Wolfgang Grodd, and Willibald Ruch. 2003. "Neural Correlates of Laughter and Humour." Brain 126(10): 2121-38.

Yue, Xiaodong, Feng Jiang, Su Lu, and Neelam Hiranandani. 2006. "To Be or Not To Be Humorous? Cross Cultural Perspectives on Humor.” Frontiers in Psychology 7: 1495. 